\title{
Decidual sialylation shows species-specific differences in the pregnant mouse and rat
}

\author{
C. J. P. Jones ${ }^{1}$, S. J. Kimber ${ }^{2}$, I. Illingworth ${ }^{2}$ and J. D. Aplin ${ }^{2,3}$ \\ ${ }^{1}$ Department of Pathological Sciences, ${ }^{2}$ School of Biological Sciences and ${ }^{3}$ Department of Obstetrics and \\ Gynaecology, University of Manchester, Manchester M13 9PT, UK
}

\begin{abstract}
Biotinylated lectins from Sambucus nigra (SNA) and Maackia amurensis (MAA), which bind to $\alpha 2,6$-linked and $\alpha 2,3$-linked sialyl residues, respectively, were used as probes to study glycan terminal modifications associated with decidualization in the uterine stroma of pregnant rats and mice. Binding of lectins from Erythrina cristagalli (ECA), Phaseolus vulgaris (leukoagglutinin, L-PHA), Triticum vulgaris (WGA) and Bandeiraea simplicifolia (BSA-1B ${ }_{4}$ ) was also examined. Tissues from rats between day 5 and day 8 of gestation and mice between day 5 and day 7 of gestation were fixed in Bouin's solution and embedded in wax prior to lectin histochemistry. On day 7 in rats and day 6 in mice, there was a marked reduction in the binding of SNA in the subluminal decidua surrounding the implantation site. In rats, MAA binding to enlarged decidual cells around the implantation chamber was increased markedly, but there was no change in mice. In both species there was de novo binding of ECA in the SNA-negative area, suggesting that the loss of $\alpha 2,6$-linked sialyl residues unmasks terminal $\mathrm{N}$-acetyl lactosamine. These findings are consistent with previous evidence of a close structural and functional similarity between the artificially induced deciduoma and true decidua of rats and show identical changes to the glycosylation patterns previously found in differentiating rat deciduoma. In both species, therefore, decidua exhibits regionally specific terminal glycosylation. However, the species-specific expression of $\alpha 2,3$-linked sialyl residues suggests distinct patterns of steroidally modulated sialyl transferase expression.
\end{abstract}

\section{Introduction}

In rodents, implantation of embryos is accompanied by marked changes in the morphology and metabolism of endometrial stromal cells, which become enlarged and polyploid with a concomitant reduction in extracellular space. This transformation is known as the decidual cell reaction, or decidualization. It can also be induced by artificial stimuli to produce a response called a deciduoma that seems to be both morphologically and biochemically identical to the decidua found in pregnant animals (O'Shea et al., 1983; Welsh and Enders, 1985; Glasser et al., 1987). Lectin histochemistry was used to examine glycosylation changes during decidualization, and striking changes in the distribution of sialyl residues in the differentiating deciduoma of the rat were demonstrated (Jones et al., 1993). Alterations were also seen in non-reducing terminal residues recognized by Erythrina cristagalli lectin (ECA) and in $\mathrm{N}$-linked glycans bound by Phaseolus vulgaris (leukoagglutinin, L-PHA). These findings suggest alterations in glycoprotein biosynthesis and sialyl transferase activity as the stromal cells differentiate. In this study, we investigated whether similar changes occur in pregnancy in rats and mice or if the presence of the embryo modifies this pattern. Illustrative

Received 19 July 1995. comparisons are presented of approximately similar stages in the development of the two species: rats at day 6 with mice at day 5 , rats at day 7 with mice at day 6 , and rats at day 8 with mice at day 7 , as development in rats is $12 \mathrm{~h}$ or more behind that of mice by the peri-implantation period.

\section{Materials and Methods}

Mice and rats

Nine MFI mice and seven Sprague-Dawley rats were obtained from Harlan Olac (Bicester, UK). For pregnant animals, females were housed individually with males of their own strain and mating was established by checking for a vaginal plug (mice) or spermatozoa in vaginal smears (rats). Pregnancy was confirmed either by flushirg embryos from part of one uterine horn on day 5 of pregnancy or by identification of decidual swellings on later days. Mice were killed by cervical dislocation and rats by chloroform anaesthetic overdose, after which the uterine horns were dissected and cut into $4-6$ pieces or individual decidual swellings where these were visible. They were fixed in Bouin's fluid overnight at $4^{\circ} \mathrm{C}$, dehydrated through a graded series of alcohols and, after clearing in xylene, embedded in paraffin wax. Sections, 5-7 $\mu \mathrm{m}$ thick, were cut, mounted on slides and dried at $60^{\circ} \mathrm{C}$. 
Table 1. Lectins used in this study and their major specificities

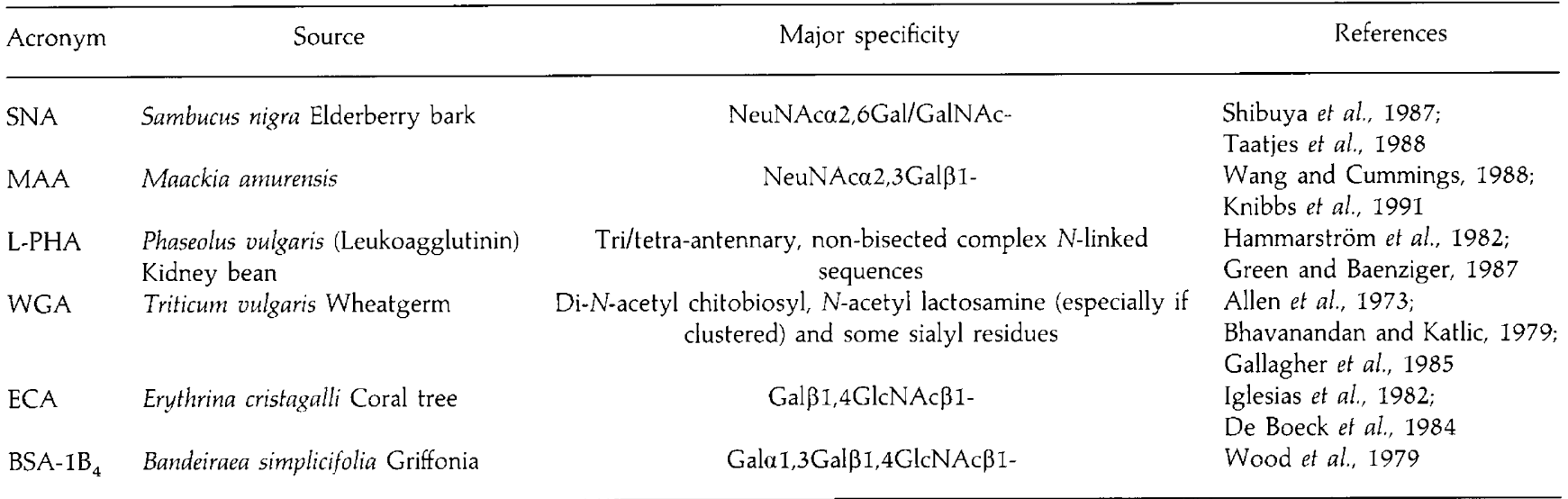

\section{Lectin histochemistry}

Staining was carried out using the method of Jones et al. (1992) with a carefully selected panel of lectins (Sigma, except for SNA and MAA which were from Boehringer-Mannheim, Lewes, UK; see Table I for their major sugar specificities). Sections were dewaxed in xylene, rinsed in absolute ethanol, and treated for $30 \mathrm{~min}$ with absolute methanol containing $0.4 \%$ $(\mathrm{v} / \mathrm{v}) \mathrm{HCl}$ and $0.5 \%(\mathrm{v} / \mathrm{v})$ hydrogen peroxide (BDH, Poole, UK) to inactivate endogenous peroxidase. After rinsing in water and washing in Tris-buffered saline, $\mathrm{pH} 7.6$ (50 mmol Tris- $\mathrm{HCl}$ $\mathrm{l}^{-1}, 0.15 \mathrm{~mol} \mathrm{NaCl} \mathrm{l}^{-1}$, TBS) for $15 \mathrm{~min}$ (three changes), sections were incubated for $30 \mathrm{~min}$ at room temperature with $10 \mu \mathrm{g}$ biotinylated lectin $\mathrm{ml}^{-1}$ in TBS plus $1 \mathrm{mmol} \mathrm{CaCl} \mathrm{l}^{-1}$, $\mathrm{pH} 7.6$ (TBSC). Washing for $15 \mathrm{~min}$ in TBSC (three changes) was followed by incubation in $5 \mu \mathrm{g}$ avidin-peroxidase $\mathrm{ml}^{-1}$ (Sigma) in $0.125 \mathrm{~mol}$ Tris- $\mathrm{HCl} \mathrm{l}^{-1}, \mathrm{pH} 7.6,0.347 \mathrm{~mol} \mathrm{NaCl}$ $1^{-1}$ (Jones et al., 1987) at room temperature for $I \mathrm{~h}$; then sections were washed in three changes of TBS over $15 \mathrm{~min}$. Sites of lectin binding were visualized using $0.05 \%(\mathrm{w} / \mathrm{v})$ diaminobenzidine tetrahydrochloride dihydrate (Aldrich Chemical Co., Gillingham) in TBS with $0.015 \%$ (v/v) hydrogen peroxide for $5 \mathrm{~min}$ at room temperature. Sections were then washed, counterstained with methyl green, dehydrated, cleared and mounted in XAM (neutral synthetic mounting medium; Gurr, BDH).

As a negative control, sections were incubated in TBSC in place of the lectin. ECA was incubated in the presence of 0.2 mol galactose $l^{-1}$ while no simple sugars were available to compete for the binding of L-PHA. As competing sugars would alter the acidity or ionic strength of sialic acid-binding lectins, SNA, MAA and WGA were controlled by incubation of $0.1 \mathrm{iu}$ neuraminidase $\mathrm{ml}^{-1}$ (Type VI from Clostridium perfringens,

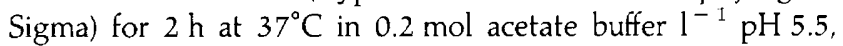
with $1 \%(\mathrm{w} / \mathrm{v})$ calcium chloride to cleave off terminal sialic acid residues. Sections of mouse (day 6) and rat (day 7) uterus were also stained with $B S A-B_{4}$ to identify trophoblast giant cells which have been reported to bind this lectin in mice (Azuma et al., 1991). All observations were made using a minimum of two implantation sites from two different mice or rats for each lectin on each day.

\section{Results}

To provide a direct comparison with the deciduoma model used by Jones et al. (1993), we used a similar panel of lectins and equivalent stages in decidual differentiation. Descriptions of lectin binding are mainly restricted to the resident stromal cells of the endometrium and their associated extracellular matrix to give a general overview of the patterns of lectin staining; binding of glandular and luminal epithelial cells, and capillary endothelium is described only where evident in the figures. Staining of the embryo will form the subject of a future report. The results have been summarized in Table 2, which shows the representative lectin binding patterns of rat and mouse decidua, in regions proximal and distal to the implantation site.

\section{Rats at day 5 of pregnancy}

Beneath the highly folded uterine lumen, there was strong membrane staining of anti-mesometrial subluminal stromal cells with SNA, and some cytoplasmic staining (Fig. 1a). Distal to the lumen, strongly staining cells were more scattered and showed up darkly against a pale background of extracellular matrix. Tissue in the subluminal area bound MAA (Fig. Ib) and L-PHA faintly, mainly to the cell membranes. Apart from occasional, isolated cells, there was virtually no binding of MAA to the stroma. With ECA, these isolated cells stained strongly (Fig. IC) and there was also a diffuse, speckled staining of the stroma, especially in peripheral regions. L-PHA showed some cytoplasmic and extracellular staining, especially in the peripheral stroma adjacent to the myometrium. WGA showed very strong membranous and cytoplasmic staining in the mesometrial subluminal area, and weaker binding to the extracellular matrix and scattered stromal cells more peripherally in the endometrium.

\section{Mice at day 5 of pregnancy}

With SNA, intense binding was present throughout the endometrium (Fig. Id), especially in the cytoplasm of some stromal cells, with a slight diminution of cytoplasmic staining 
Table 2. Lectin-binding patterns of rat and mouse decidua, proximal and distal to the implantation site (excluding capillaries, glands and endothelia)

\begin{tabular}{|c|c|c|c|c|c|c|c|c|}
\hline Lectin & Proximal & Distal & Proximal & Distal & Proximal & Distal & Proximal & Distal \\
\hline & \multicolumn{2}{|c|}{ Day 5} & \multicolumn{2}{|c|}{ Day 6} & \multicolumn{2}{|c|}{ Day 7} & \multicolumn{2}{|c|}{ Day 8} \\
\hline \multicolumn{9}{|l|}{ Rat } \\
\hline SNA & $2-3$ & $1-2$ & $2-3$ & $2-3$ & $0-1$ & 3 & $1-2$ & $3-4$ \\
\hline MAA & 1 & 1 & 1 & 1 & $0-1$ & $0-1$ & 3 & $1-2$ \\
\hline L-PHA & 1 & $1-2$ & $0-1$ & $0-1$ & $0-1$ & $0-1$ & 3 & 1 \\
\hline WGA & $2-3$ & $2-3$ & $2-3$ & $2-3$ & 3 & $2-3$ & $3-4$ & 3 \\
\hline $\mathrm{ECA}$ & $1-2$ & $1-2$ & $0-1$ & $0-1$ & $0-1$ & $1-2$ & $2-3$ & $I$ \\
\hline Mouse & & & \multicolumn{2}{|c|}{ Day 5} & \multicolumn{2}{|c|}{ Day 6} & \multicolumn{2}{|c|}{ Day 7} \\
\hline SNA & & & $3-4$ & 4 & 1-2 & 4 & 1-2 & $3-4$ \\
\hline MAA & & & 2 & 2 & 2 & 2 & 2 & 2 \\
\hline L-PHA & & & $2-3$ & 2 & $1-2$ & $1-2$ & $3-4$ & 1 \\
\hline WGA & & & $3-4$ & $3-4$ & $3-4$ & $3-4$ & $3-4$ & $3-4$ \\
\hline $\mathrm{ECA}$ & & & $2-3$ & 2 & 3 & $1-2$ & $3-4$ & 2 \\
\hline
\end{tabular}

Key to staining intensities: 0 : negative; 1 : weak; 2 : moderate; 3 : strong; 4 : intense. The figures in bold show that in rats both SNA and MAA change on days 7 and 8 , whereas in mice only the SNA changes.

around the uterine lumen, where staining was mainly pericellular. MAA staining was faint and uniform throughout the endometrium (Fig. 1e), with a stippled appearance in and around the cells and some membranous staining. Scattered individual cells also bound this lectin strongly. With L-PHA, there was light to moderate staining throughout the endometrium that was mainly granular but with some binding to the cell membranes; the staining reaction was marginally stronger around the antimesometrial uterine lumen. There was also membranous and cytoplasmic staining of the scattered cell population. WGA stained uniformly and strongly throughout the endometrium, with individual scattered cells staining particularly intensely. ECA showed faint to moderate binding (Fig. 1f), increasing slightly near the uterine lumen, especially antimesometrially adjacent to the implanting embryo, with strong staining of the scattered cells.

\section{Rats at day 6 of pregnancy}

The uterine lumen exhibited a smooth, oval profile, and surrounding stromal cells were variable in their binding of SNA. Membrane staining was less prominent than before. There was little change in MAA binding, while L-PHA showed diminished staining intensity, especially near the myometrium. WGA bound uniformly throughout the bulk of the stroma, apart from some variable staining of subluminal cells and strong binding to scattered stromal cells. ECA also bound to a distinct population of widely dispersed cells, but showed diminished binding elsewhere in the stroma.

\section{Rats at day 7 of pregnancy}

By this stage, the uterine lumen was narrow and slit-like at the implantation site, with the embryo in contact with both lateral walls. Staining with SNA was altered markedly (Fig. 2a), with a more or less complete loss of binding to approximately six layers of cells adjacent to the implantation chamber (Fig. 2b). Peripherally, SNA staining was moderate to strong. MAA showed little change (Fig. 2c), and there was virtually no staining with L-PHA (Fig. 3a). A tongue of increased binding extending from the antimesometrial tip of the decidual crypt was prominent with WGA (Fig. 3b), and strong staining with this lectin extended back along the sides of the implantation chamber, delineating an area more or less equivalent to that showing diminished SNA binding. The rest of the stroma was stained with moderate intensity, increasing slightly at the border with the myometrium, and individual cells showed a range of binding intensity. The staining with ECA showed no change from day 6 (Fig. $3 \mathrm{c}$ ), while $\mathrm{BSA}^{-1 \mathrm{~B}_{4}}$ did not bind to trophoblast giant cells.

\section{Mice at day 6 of pregnancy}

There was a marked reduction in binding of SNA in the primary decidual zone except for a narrow band adjacent to the implanting embryo and the epithelial remnants in the decidual crypt (Fig. 2d). More peripherally, adjacent to the myometrium and in the interimplantation sites, strong cytoplasmic and extracellular matrix staining was still present. MAA, however, showed little change in expression over most of the decidua (Fig. 2e). Slightly stronger MAA staining was found in a narrow band, 5-6 cells in depth, around the implantation site including decidual cells and trophoblast giant cells. Beneath this band, MAA staining was prominent in maternal capillaries (Fig. 2f). Trophoblast giant cells also bound $\mathrm{BSA}-1 \mathrm{~B}_{4}$ (Fig. $2 \mathrm{~F}$, inset). With L-PHA, there was again a ring of strong staining immediately around the implantation chamber in the primary trophoblast giant cells (Fig. 3d) and 

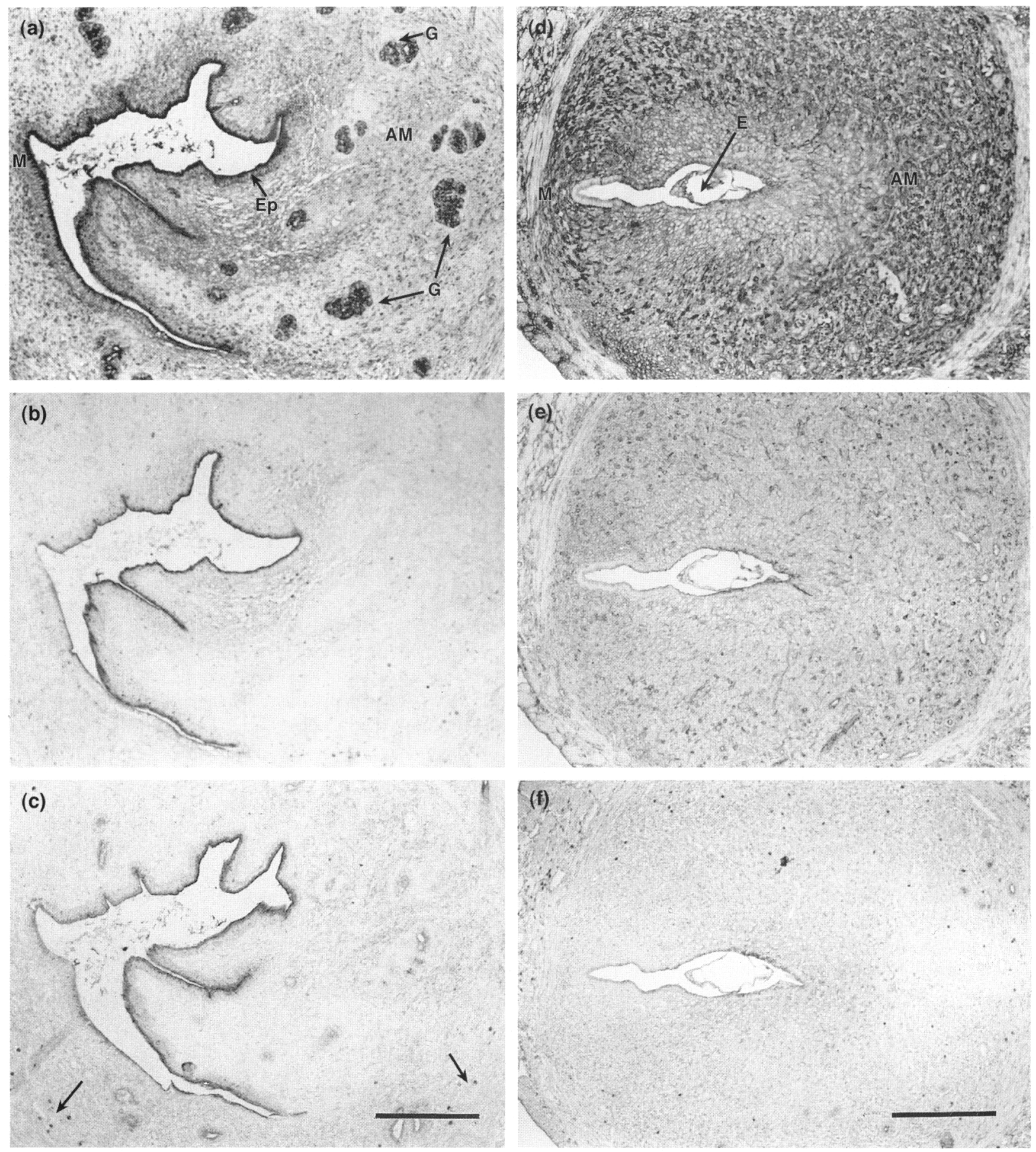

Fig. 1. Lectin histochemistry of rat $(a-c)$ and mouse $(d-f)$ uterus on day 5 of pregnancy. (a) Lectin from Sambucus nigra (SNA) binds mainly to the area under the uterine lumen. The glands $(G)$ and uterine epithelium (Ep) also stain strongly. (b) There is only faint binding of the lectin from Maackia amurensis (MAA). (c) The lectin from Erythrina cristagalli (ECA) stains in a speckled fashion, with strong staining of occasional scattered cells near the myometrium (arrows). (d) There is strong staining by SNA with a slight subluminal dimunition of binding. Part of the embryo (E) in its implantation chamber can be seen. (e) Binding with MAA is faint and uniform throughout the endometrium. (f) There is faint binding of ECA, increasing slightly near the uterine lumen, antimesometrially. (AM: antimesometrial; M: mesometrial pole of uterus.) Scale bars represent $250 \mu \mathrm{m}$.

decidual capillary endothelium was also prominently stained. Beyond this, L-PHA showed decreased binding around the implantation area with little granularity evident, apart from occasional darkly stained cell processes. The binding of WGA (Fig. 3e) continued to be uniform and strong throughout the endometrium, with intense staining around the implantation chamber, including binding by the embryo and trophoblast giant cells. With ECA, there was a wide area of moderate to strong binding to decidual cells extending from the implantation site to midway across the endometrium. Staining was 

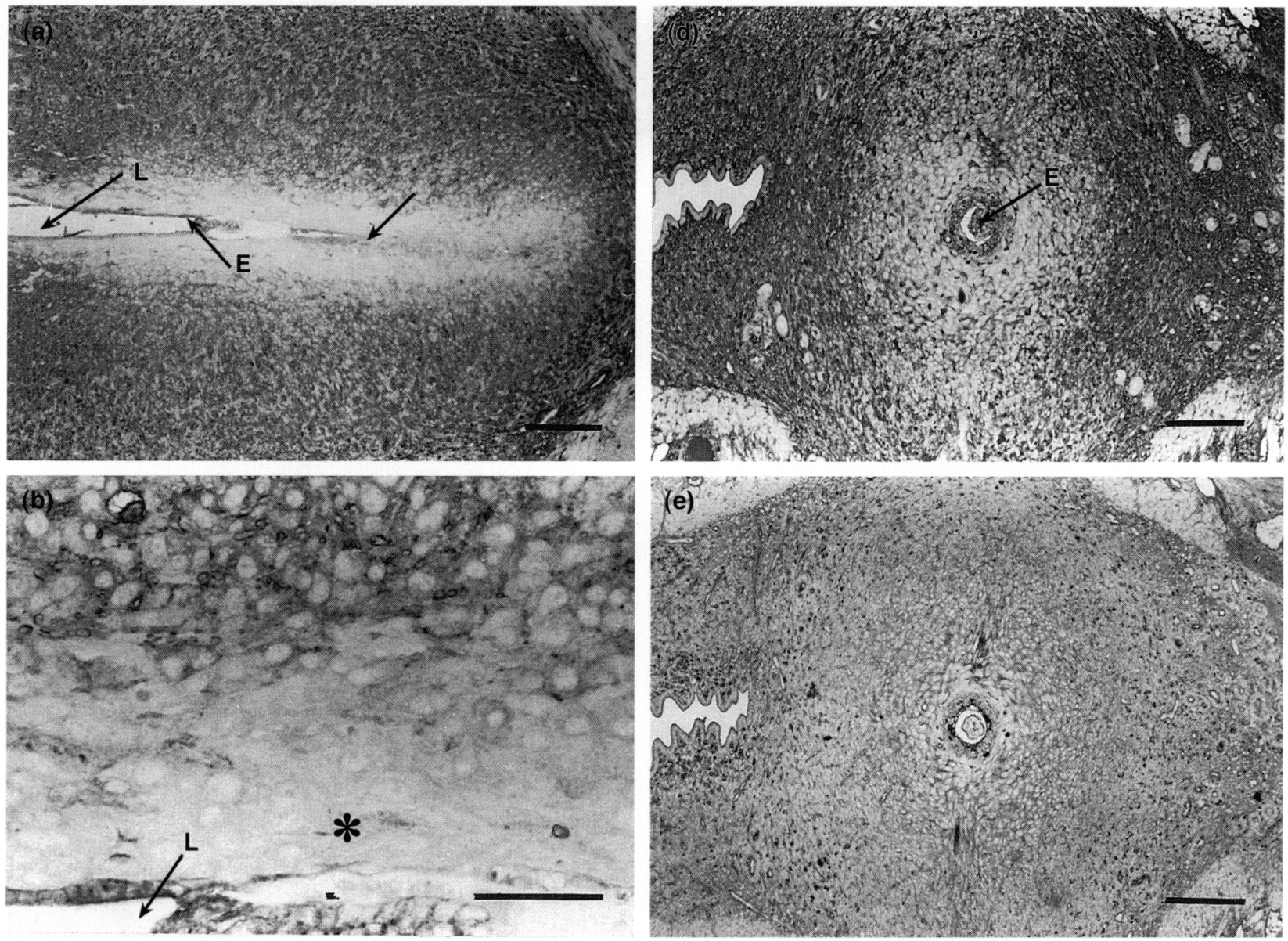

(c)

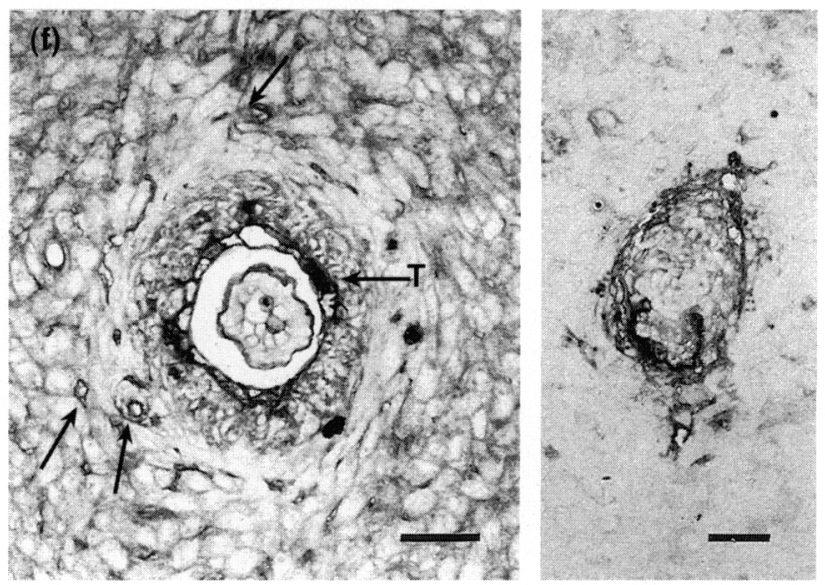

Fig. 2. Lectin histochemistry of uterus of rat on day 7 (a-c) and mouse on day $6(d-f)$ of pregnancy. (a) There is a marked reduction in the binding of Sambucus nigra (SNA) around the uterine lumen (L). Part of the embryo (E) can be seen. Only a narrow tongue of cells extending from the antimesometrial tip of the decidual crypt exhibits faint binding (arrow). (b) Higher power view of part of the uterine lumen (L) and area of decidua showing loss of SNA binding $\left(^{*}\right)$. (c) There is little change in the binding of MAA. (d) In this transverse section, the reduction in the binding of SNA can be seen clearly. The embryo (E) and area immediately adjacent to the implantation site are well-stained. (e) There is little change in the binding of Maackia amurensis (MAA) over most of the endometrium. (f) In this higher power view, strong staining of the trophoblast giant cells $(T)$ and surrounding capillaries (arrows) with MAA can be seen. Inset: the lectin from Bandeiraea simplicifolia-1B $B_{4}\left(B S A-1 B_{4}\right)$ binds strongly to the trophoblast giant cells. Scale bars represents $200 \mu \mathrm{m}(a, c, d$ and $e)$ and $50 \mu \mathrm{m}(b$ and $\mathrm{f}$ ).

strong adjacent to the implantation chamber (Fig. 3f), while adjacent to the myometrium it was faint. Isolated cells stained darkly with L-PHA, WGA and ECA were mainly restricted to an area adjoining the myometrium.
Rats at day 8 of pregnancy

The area of diminished SNA binding was now extended considerably (Fig. 4a), comprising most of the large cells of the 
(a)
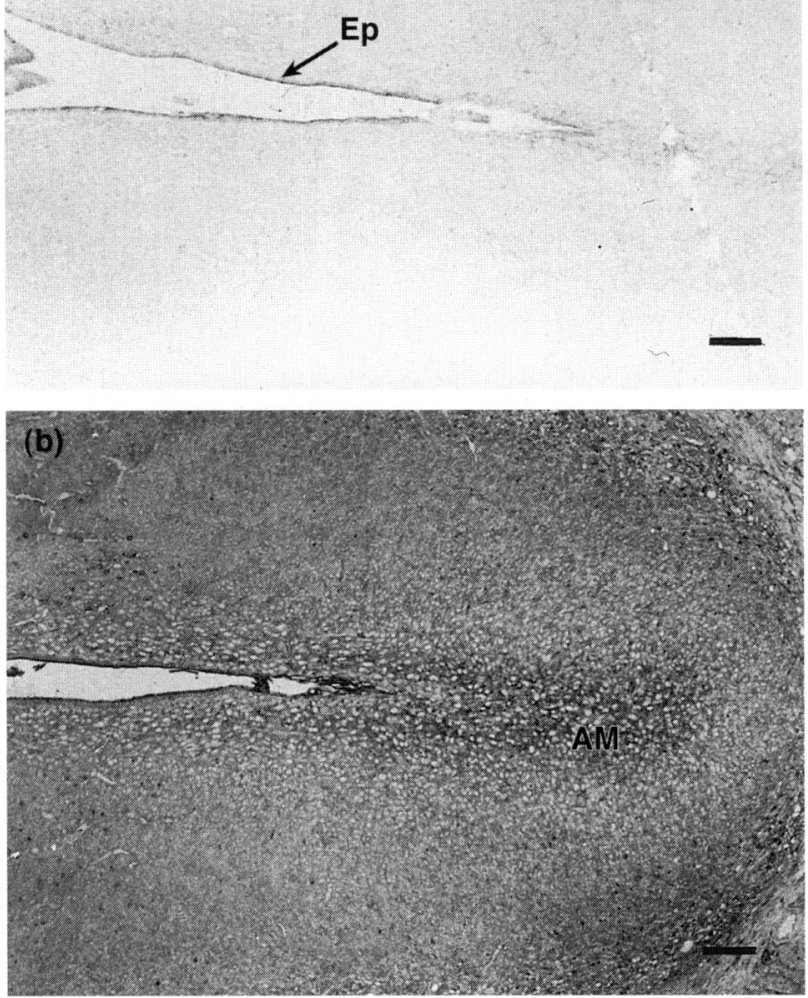

(c)

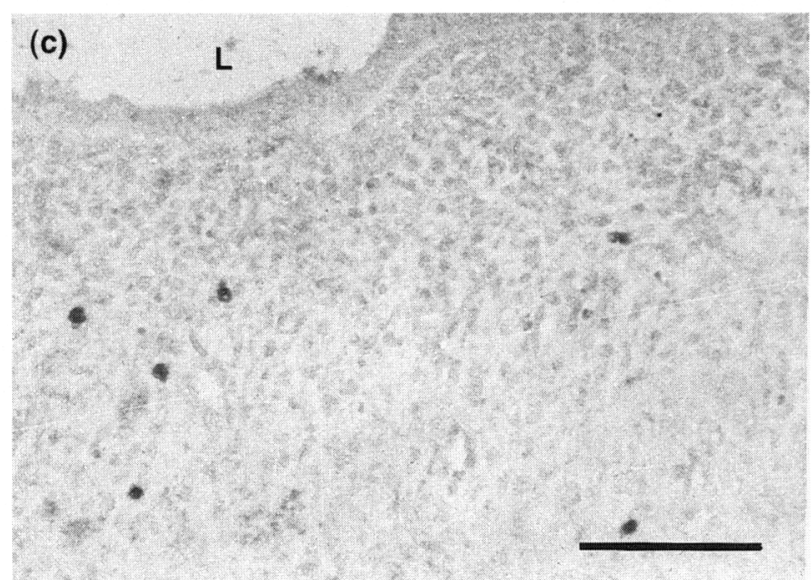

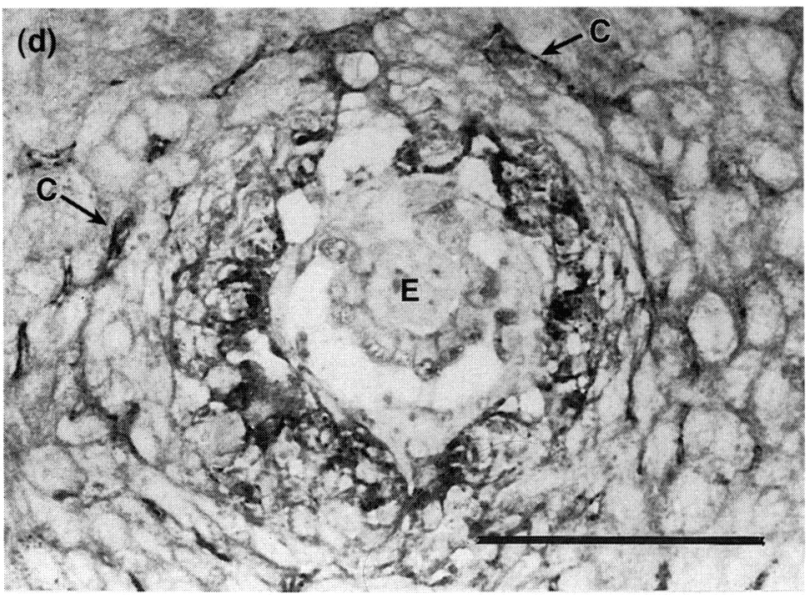
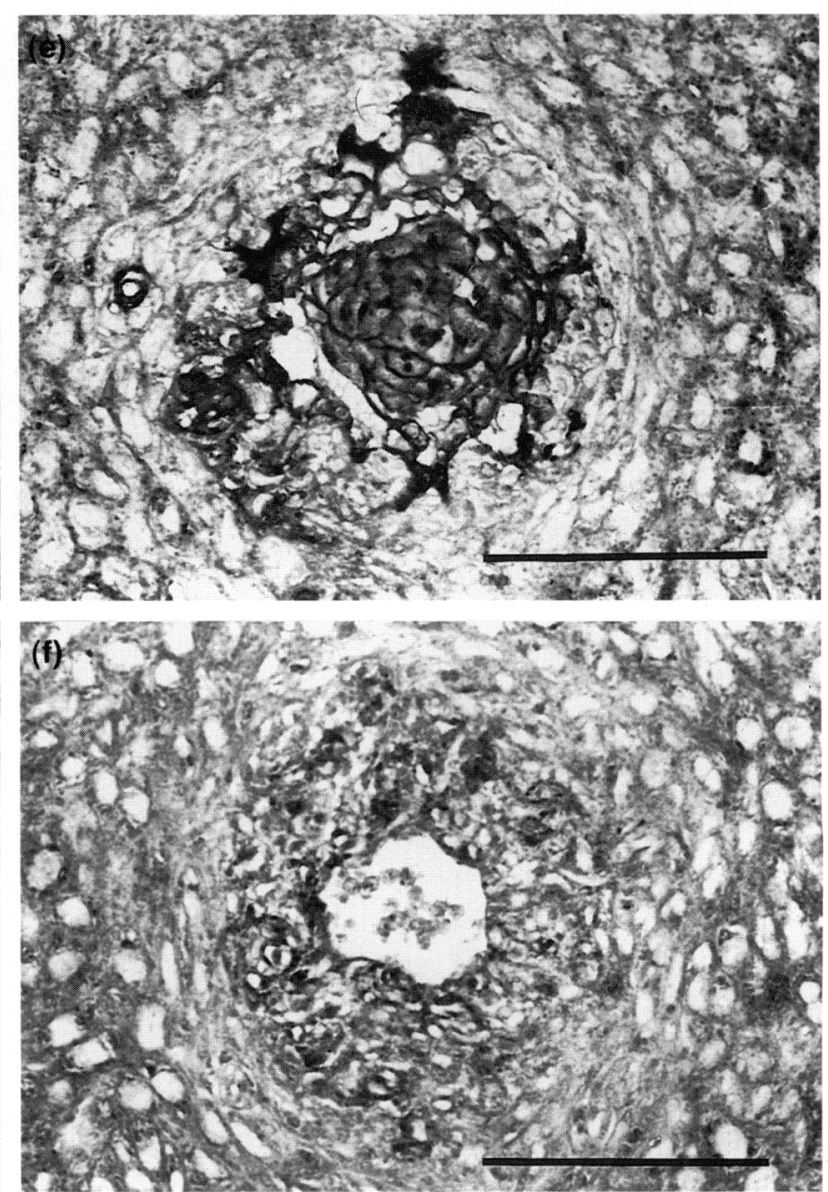

Fig. 3. Lectin histochemistry of uterus of rat on day $7(\mathrm{a}-\mathrm{c})$ and mouse on day $6(\mathrm{~d}-\mathrm{f})$ of pregnancy. (a) Apart from some binding by the luminal epithelium (Ep), there is virtually no staining with the lectin from Phaseolus vulgaris (leukoagglutinin) (L-PHA). (b) The lectin from Triticum vulgaris (WGA) stains strongly, especially the luminal epithelium and in the antimesometrial region beneath the lumen (AM), extending back along the sides of the implantation chamber. (c) There is strong staining of scattered cells with Erythrina cristagalli (ECA). The rest of the stroma binds only weakly. Part of the lumen (L) is visible. (d) With L-PHA, there is strong staining around the implantation chamber and of the capillary endothelium $(\mathrm{C})$; part of the embryo (E) can be seen. (e) WGA stains the trophoblast giant cells and embryo particularly intensely. (f) ECA binds around the implantation chamber, with moderate staining of the decidual cells. Scale bars represent $100 \mu \mathrm{m}$.

decidual zone adjacent to the implantation chamber and decidual crypt. These showed only very faint binding of SNA, mainly at their surfaces, in contrast to the deeper layers, which bound SNA strongly. In the antimesometrial decidual crypt, trophoblast showed moderate staining, while maternal capillary endothelium also stained strongly. With MAA, the decidual cells around the implantation chamber showed intense binding (Fig. 4b), with intracellular stippling and strong pericellular 

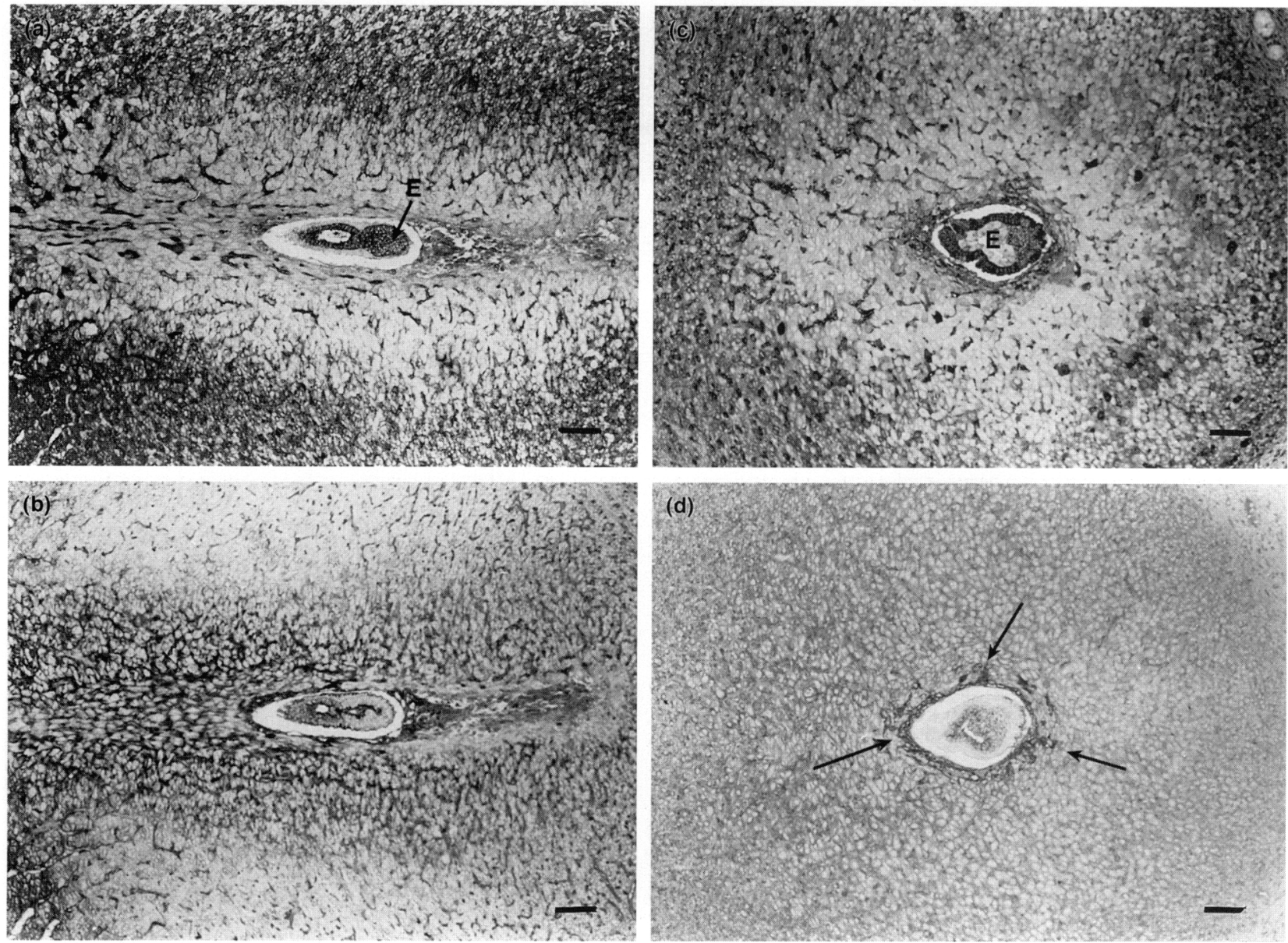

Fig. 4. Lectin histochemistry of uterus of rat on day 8 (a-b) and mouse on day 7 (c-d) of pregnancy. (a) The area of diminished binding with Sambucus nigra (SNA) has widened compared with day 7, although vessels and the embryo (E) now stain strongly. (b) There is now intense staining of the decidual cells around the implantation chamber with Maackia amurensis (MAA). (c) With SNA, there is now an extensive zone of reduced binding by decidual cells around the implantation site, although capillaries stain strongly. Part of the embryo (E) can be seen. (d) Staining with MAA is pale and uniform apart from the primary trophoblast giant cells (arrows). Scale bars represent $100 \mu \mathrm{m}$.

staining. Towards the mesometrial pole, the column of positively-stained cells fanned out away from the decidual zone. The decidual crypt was also stained, although it was enclosed by a narrow sheath that showed less binding. The endometrium midway between the implantation chamber and myometrium, on the lateral aspects, was only faintly stained, with a deepening in intensity near the myometrium. There was intense membranous and cytoplasmic staining of a population of cells that clustered around the mesometrial remnant of the lumen. L-PHA showed intense pericellular staining and some intracellular stippling of the enlarged decidual cells (Fig. 5a). There was strong binding to cells within the decidual crypt and around the implantation chamber. Mesometrially, there was less fanning out of the column of positively stained cells than with MAA and the cells around the mesometrial remnant generally lacked staining of the surface membrane. More peripheral areas of the endometrium were almost unstained, apart from the mesometrial pole. WGA showed strong binding throughout the endometrium (Fig. 5b), particularly around the primary decidual zone of the implantation chamber and antimesometrial decidual crypt. The staining pattern echoed that of
MAA, although a more extensive area showed intense binding; this was more prominent near the embryo, where it was both pericellular and more faintly cytoplasmic. More peripherally, it was mainly cytoplasmic. The population of cells around the mesometrial remnant was again strongly stained with this lectin. There was a marked increase in the binding of ECA (Fig. 5c), which now showed a light to moderate staining of the primary decidual cells that was more intense towards the antimesometrial pole. The decidual cells had pale, diffuse cytoplasmic staining decreasing towards the myometrium. Only faint binding was present over the rest of the endometrium, and the population of cells that bound MAA and L-PHA intensely were not stained by this lectin.

\section{Mice at day 7 of pregnancy}

At day 7 there was an extensive zone of reduced binding of SNA around the implantation site, except for a narrow strip of cells adjacent to the implantation chamber (Fig. 4c). Invading trophoblast cells in this region were stained as well as 

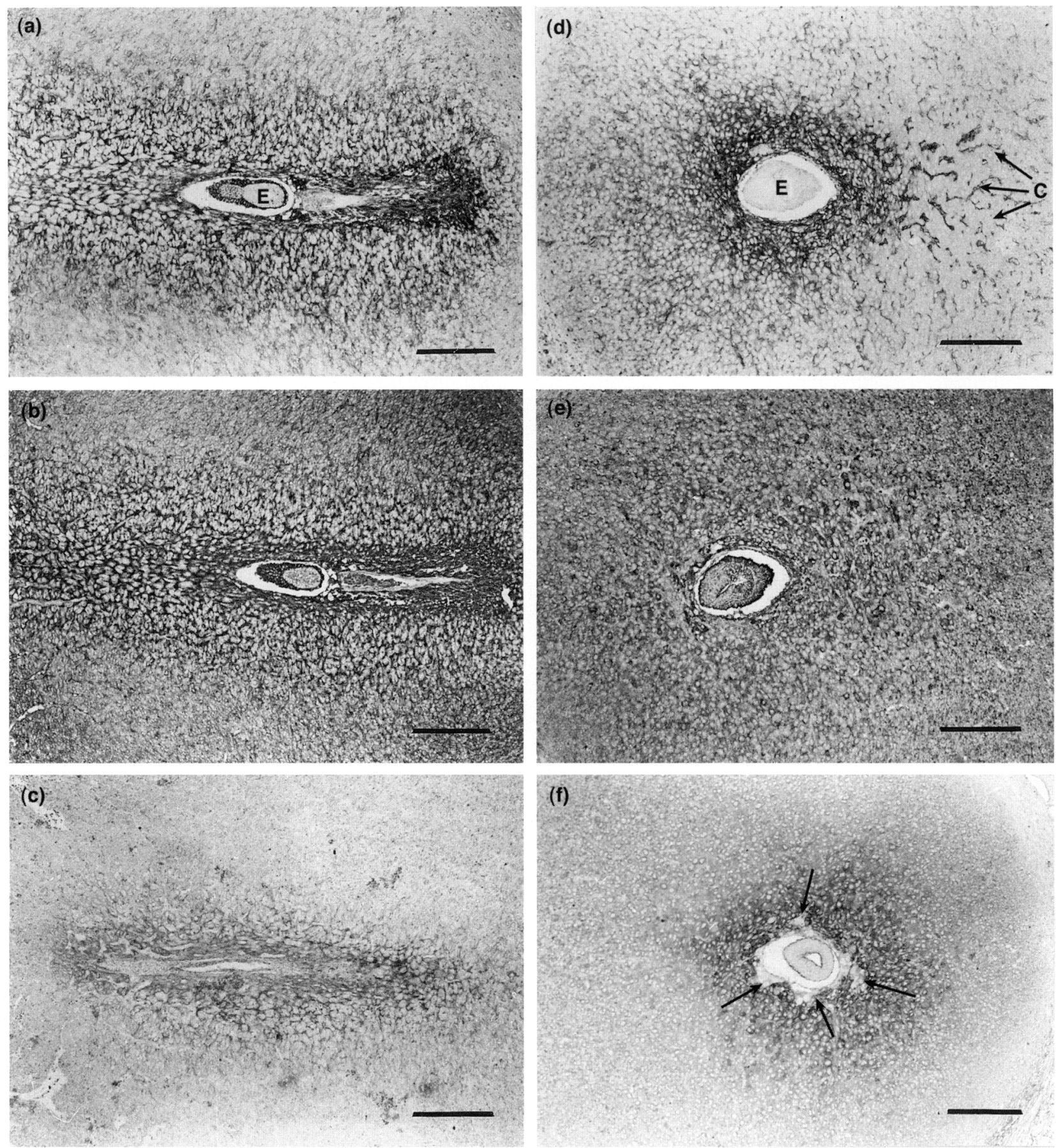

Fig. 5. Lectin histochemistry of uterus of rat on day $8(a-c)$ and mouse on day $7(d-f)$ of pregnancy. (a) There is strong staining with Phaseolus vulgaris (leukoagglutinin) (L-PHA) mainly around the implantation chamber and the embryo (E). (b) Triticum vulgaris (WGA) binds strongly, especially around the primary decidual zone of the implantation chamber. (c) There is a marked increase in the binding of Erythina cristagalli (ECA) to the primary decidual cells around the implantation chamber. (d) L-PHA shows intense binding to the area adjacent to the implantation chamber and to surrounding capillaries (C). The embryo (E) is unstained. (e) There is uniform, strong binding of WGA throughout the endometrium. (f) There is increased binding of ECA around the implantation chamber apart from unstained trophoblast giant cells (arrows). Scale bars represent $250 \mu \mathrm{m}$.

superficial maternal capillary endothelium. More peripherally, a population of decidual cells retained intense SNA binding, producing a patchwork effect. Near the myometrium, where cells were smaller, staining intensity was again high. With MAA, staining was relatively pale and uniform (Fig. 4d) apart from the cells adjacent to the implantation chamber, where intense binding was seen on primary trophoblast giant cells. Certain scattered cells again bound MAA, but at this stage they were less conspicuous. L-PHA showed intense cytoplasmic and pericellular staining adjacent to the implantation 
chamber (Fig. 5d). This binding decreased gradually with increasing distance from the implantation chamber. The area of intense L-PHA staining was narrower than that showing decreased SNA-binding activity (compare with Fig. 4c). L-PHA also stained maternal capillary endothelium. The pattern of uniform WGA binding was similar to that seen on day 6 (Fig. 5e). ECA bound strongly to the cytoplasm and cell surfaces of decidual cells around the decidual crypt and implantation site (Fig. 5f), apart from an area immediately adjacent to the implantation site. In this region, trophoblast giant cells were poorly stained in contrast to their strong staining with SNA and MAA (Fig. $4 \mathrm{c}$ and d). More peripherally, the area of intense staining decreased gradually and became mainly cytoplasmic. The overall intensity of staining was, however, slightly greater than seen before.

\section{Discussion}

These results demonstrate that stage- and regionally-specific glycosylation is a feature of decidual cells in both rats and mice. This is effected under maternal control, as shown by similar findings in artificially induced deciduoma (Jones et al., 1993). The loss during decidual differentiation of terminal $\alpha 2,6$-linked sialic acid, recognized by SNA, is consistent in both species. In rats, it is accompanied by upregulation of a2,3-linked sialyl residues recognized by MAA. The observed increase in binding of WGA is probably also accounted for by $\alpha 2,3$-linked sialic acid terminals (Kronis and Carver, 1982). Increased binding of L-PHA and ECA are also evident. In mice, ECA and L-PHA binding are increased in decidual cells but MAA binding remains unaltered except in a narrow band of 5-6 cells adjacent to the implantation chamber. Thus, it appears that alterations in chain capping sugars occur in both species. The diminished abundance of $\alpha 2,6$-linked sialic acid may expose new subterminal residues, such as the $\mathrm{N}$-acetyl lactosamines that act as ligands for ECA (and WGA). In rats (but not mice), these in turn may act as substrates for addition of $\alpha 2,3$-linked sialyl residues. Increased amounts of tri/tetra-antennary, non-bisected complex $\mathrm{N}$-linked glycan are synthesized in the subluminal stroma in both pregnant rats and mice, as shown by the increased binding of L-PHA. Terminaily fucosylated type I and type II chains do not appear to be expressed in mouse (Kimber et al., 1988; Kimber and Lindenberg, 1990) or rat (Kimber et al., 1995) stroma or decidua.

It has been shown that steroid hormones have a marked stimulatory effect on glycoprotein biosynthesis, particularly with respect to $N$-linked structures and lactosaminoglycans (Dutt et al., 1988; Carson et al., 1990). The present data suggest significant changes in the activity of specific glycosyltransferases, with both similarities and differences between the two species. However, further studies will be required to investigate the mechanisms involved in effecting the observed changes in lectin binding: altered glycosylation of existing components, the production of new glycoconjugates, or both.

Altered glycosylation in decidua may be related to the remodelling of the extracellular matrix (Kleinfeld et al., 1976; Parr et al., 1986; Mulholland and Jones, 1993; Mulholland et al,
1992) and the appearance of electron-dense flocculent material between the enlarging cells. Laminin, which is $\mathrm{N}$-glycosylated, has also been found to increase during days 6 and 7 of pregnancy in rats (Glasser et al., 1987; Clark et al., 1993), and this may be related to the increase in L-PHA staining at this time. In addition, decidualizing stromal cells produce new cell surface structures, including adherens and gap junctions (Kleinfeld et al, 1976; Brokelman and Biggers, 1979; O'Shea et al., 1983; Parr ef al., 1986; Mulholland and Jones, 1993; Winterhager et al., 1993). Thus, decreased binding of SNA (with a possible reduction of coulombic repulsion) may be related to the development of intercellular contacts. It will be interesting to examine the localization of the newly expressed glycodeterminants at the ultrastructural level and to characterize the macromolecules with which they are associated.

Mouse primary giant cells, identified by staining with BSA-1B ${ }_{4}$ (Azuma et al., 1991), react with lectins that bind both $\alpha 2,3$ and $\alpha 2,6$-sialyl residues. Trophectoderm of the preimplantation blastocyst has been demonstrated to express $\alpha 2,3$ and poly- $\alpha 2,8$, but not $\alpha 2,6$-linked, sialyl residues (Shevinsky et al., 1982; Kimber et al., 1993, 1994; S. J. Kimber, unpublished). In contrast to our results with SNA and MAA, expression of poly- $\alpha 2,8$ sialic acid is downregulated on mouse trophoblast at the time of implantation (Kimber et al., 1994). However, an antibody that recognizes $\alpha 2,3$ sialyl residues found in brain gangliosides did not react with trophectoderm of day 5-7 mouse embryos (Pennington et al., 1985).

Sialic acids may be functionally important in specific recognition events (as in selectin action), in increasing the net negative charge of the cell surface, which may in turn increase intercellular spaces, and in masking of subterminal glycan recognition structures. Changes in the amount, type or linkage of sialic acid in tumour cell glycoconjugates can affect tumour growth and metastasis (Takano et al., 1994). Removal of $\alpha 2,6$-linked sialic acid may be important in the preparation of the endometrium for trophoblast invasion in these species. Cell surface sialic acid residues regulate cell adhesiveness negatively in a number of cell lines (Shimamura et al., 1994). Increased SNA staining has been associated with the loss in cell surface adhesiveness that occurs in malignancy. In a study of colonic tumours, Sata et al. (1991) found that an increase in $\alpha 2,6$-linked sialic acid was associated with the histological features of malignancy, while $\alpha 2,3$-linked residues progressively decreased with the degree of dysplasia in colonic adenoma, although both types of residue were found in carcinoma. Similar effects have been seen in a study of ulcerative colitis (McMahon et al., 1994), with increased staining of SNA in all grades of the disease. Smaller changes were evident with MAA, although binding was lost in severe forms of the disease. Given possible parallels between trophoblast and tumour cells (Kirby, 1965; Lala and Graham, 1990; Graham and Lala, 1992), it will be interesting to examine the effect of modulating trophoblast cell surface sialic acid on blastocyst attachment and outgrowth in vitro. In contrast, an increase in intercellular contact and adhesiveness occurs in the maternal stroma during decidualization. Changes in decidual glycosylation must, therefore, be considered in relation both to the local function and integrity of the maternal tissue and its interaction with the developing placenta.

Downloaded from Bioscientifica.com at 04/26/2023 01:00:02PM 


\section{References}

Allen AK, Neuberger $A$ and Sharon $N$ (1973) The purification and specificity of wheat germ agglutinin Biochemical Journal 131 155-162

Azuma M, Kanai Y, Ogura A, Kurohmaru M and Hayashi Y (1991) Changes in cell surface and intracellular glycoproteins of trophoblastic giant cells during mouse placentation Histochemistry 95 54 I-548

Bhavanandan VP and Katlic AW (1979) The interaction of wheat germ agglutinin with sialoglycoproteins. The role of sialic acid Journal of Biological Chemistry 254 4000-4008

Brokelman J and Biggers JD (1979) Studies on the development of cell contacts and of the intercellular matrix during decidualisation in the rat Archiv für Gynäkologie 227 103-117

Carson DD, Farrar JD, Laidlaw J and Wright DA (1990) Selective activation of the $N$-glycosylation apparatus in uteri by estrogen Journal of Biological Chemistry 265 2947-2955

Clark DE, Hurst PR, McLennan IS and Myers DB (1993) Immunolocalization of collagen type I and laminin in the uterus on days 5 to 8 of embryo implantation in the rat Anatomical Record 237 8-20

De Boeck H, Loontiens FG, Lis $\mathbf{H}$ and Sharon N (1984) Binding of simple carbohydrates and some $\mathrm{N}$-acetyllactosamine-containing oligosaccharides to Erythrina cristagalli agglutinin as followed with a fluorescent indicator ligand Archives of Biochemistry and Biophysics 234 297-304

Dutt A, Tang J-P and Carson DD (1988) Estrogen preferentially stimulates lactosaminoglycan-containing oligosaccharide synthesis in mouse uteri Journal of Biological Chemistry 263 2270-2279

Gallagher JT, Morris A and Dexter TM (1985) Identification of two binding sites for wheat germ agglutinin on polylactosamine-type oligosaccharides Biochemical Journal 231 115-122

Glasser SR, Lampelo S, Munir MI and Julian JA (1987) Expression of desmin, laminin and fibronectin during in situ differentiation (decidualization) of rat uterine stromal cells Differentiation 35 132-142

Graham CH and Lala PK (1992) Mechanism of placental invasion of the uterus and their control Biochemistry and Cell Biology 70 867-874

Green ED and Baenziger JU (1987) Oligosaccharide specificities of Phaseolus vulgaris leukoagglutinating and erythroagglutinating phytohemagglutinins. Interactions with $\mathrm{N}$-glyconase-released oligosaccharides Journal of Biological Chemistry $26212018-12029$

Hammarström S, Hammarström ML, Sunblad G and Lonnfron J (1982) Mitogenic leukoagglutinin from Phaseolus vulgaris binds to a pentasaccharide unit in $\mathrm{N}$-acetyllactosamine-type glycoprotein glycans Proceedings of the National Academy of Sciences USA 79 1611-1615

Iglesias JL, Lis $\mathrm{H}$ and Sharon $\mathrm{N}$ (1982) Purification and properties of a v-galactose/ $N$-acetyl-1)-galactosamine-specific lectin from Erythrina cristagalli. European Journal of Biochemistry 123 247-252

Jones CIP, Mosley SM, Jeffrey IJM and Stoddart RW (1987) Elimination of the non-specific binding of avidin to tissue sections Histochemical Journal 19 264-268

Jones CJP, Morrison CA and Stoddart RW (1992) Histochemical analysis of rat testicular glycoconjugates. (1) Subsets of $N$-linked saccharides in seminiferous tubules Histochemical Journal 24 319-326

Jones CIP, Aplin JD, Mulholland J and Glasser SR (1993) Patterns of sialylation in differentiating rat decidual cells as revealed by lectin histochemistry Journal of Reproduction and Fertility 99 635-645

Kimber SJ and Lindenberg S (1990) Hormonal control of carbohydrate determinants involved in implantation Journal of Reproduction and Fertility $\mathbf{8 9}$ $13-21$

Kimber SJ, Lindenberg S and Lundblad A (1988) Distribution of some Galß1$3(4) \mathrm{GlcNAc}$-related carbohydrate antigens on the mouse uterine epithelium in relation to the peri-implantation period Journal of Reproductive Immunology 12 297-313

Kimber SJ, Brown DG, Pahlsson P and Nilsson B (1993) Carbohydrate antigen expression in murine embryonic stem cells and embryos. II. Sialylated antigens and glycolipid analysis Histochemical Journal 25 628-641

Kimber SJ, Bentley J, Ciemerych M, Moller CJ and Bock E (1994) Expression of N-CAM in fertilised pre- and periimplantation and parthenogenetically activated mouse embryos European Journal of Cell Biology 63 102-113
Kimber SJ, Illingworth IM and Glasser SR (1995) Expression of carbohydrate antigens in the rat uterus during early pregnancy and after ovariectomy and steroid replacement Journal of Reproduction and Fertility 103 75-87

Kirby DRS (1965) The 'invasiveness' of trophoblast. In The Early Conceptus, Normal and Abnormal pp 68-74. Ed. WW Park. University of St Andrews Press, Edinburgh

Kleinfeld RG, Morrow HA and DeFeo VJ (1976) Intercellular junctions between decidual cells in the growing deciduoma of the pseudopregnant rat uterus Biology of Reproduction 15 593-603

Knibbs R, Goldstein IJ, Ratcliffe RM and Shibuya N (1991) Characterization of the carbohydrate binding specificity of the leukoagglutinating lectin from Maackia amurensis Journal of Biological Chemistry 226 83-88

Kronis KA and Carver JP (1982) Specificity of isolectins of WGA for sialyloligosaccharides: a $360 \mathrm{MHz}$ proton $\mathrm{nmr}$ binding study Biochemistry 21 3050-3057

Lala PK and Graham CH (1990) Mechanisms of trophoblast invasiveness and their control: the role of proteases and protease inhibitors Cancer Metastasis Review 9 369-379

McMahon RFT, Jones CJP, Dutt S and Stoddart RW (1994) Altered oligosaccharide expression in ulcerative colitis with increasing grades of inflammation Glycosylation and Disease 1 235-245

Mulholland J and Jones CJP (1993) Characteristics of uterine aging Microscopy Research and Technique 25 148-168

Mulholland J, Aplin JD, Hong L, Ayad S and Glasser SR (1992) Loss of type VI collagen from rat endometrial stroma during decidualisation Biology of Reproduction 46 1136-1143

O'Shea JD, Kleinfeld RG and Morrow HA (1983) Ultrastructure of decidualization in the pseudopregnant rat American Journal of Anatomy 116 271-298

Parr MB, Tung HN and Parr EL (1986) The ultrastructure of the rat primary decidual zone American Journal of Anatomy 176 423-436

Pennington JE, Rastan S, Roelke D and Feizi T (1985) Saccharide structures of the mouse embryo during the first eight days of development Journal of Embryology and Experimental Morphology 90 335-361

Sata T, Roth J, Zuber C, Stamm B and Heitz PU (1991) Expression of $\alpha 2,6$-linked sialic acid residues in neoplastic but not in normal human colonic mucosa American Journal of Pathology 139 1435-1448

Shevinsky LH, Knowles BB, Damjanov I and Solter D (1982) Monoclonal antibody to murine embryos defines a stage specific embryonic antigen expressed on mouse embryos and human teratocarcinoma cells Cell $\mathbf{3 0}$ 697-705

Shibuya N, Goldstein IJ, Broekaert WF, Nsimba-Lubaki M, Peeters B and Peumans WJ (1987) The elderberry (Sambucus nigra L.) bark lectin recognizes the Neu 5 Ac $(\alpha 2,6) \mathrm{Gal} / \mathrm{GalNAc}$ sequence Journal of Biological Chemistry 262 1596-1601

Shimamura M, Shibuya N, Ito M and Yamagata T (1994) Repulsive contribution of surface sialic acid residues to cell adhesion to substratum Biochemistry and Molecular Biology International 33 871-878

Taatjes DJ, Roth J, Peumans W and Goldstein IJ (1988) Elderberry bark lectingold techniques for the detection of $\operatorname{Neu} 5 \mathrm{Ac}(\alpha 2,6) \mathrm{Gal} / \mathrm{GalNAc}$ sequences: applications and limitations Histochemical Journal 20 478-490

Takano R, Muchmore E and Dennis JW (1994) Sialylation and malignant potential in tumour cell glycosylation mutants Glycobiology 4 665-674

Wang W-C and Cummings RD (1988) The immobilised leukoagglutinin from the seeds of Maackia amurensis binds with high affinity to complex-type Asn-linked oligosaccharides containing terminal sialic acid-linked $\alpha 2,3$ to penultimate galactose residues Journal of Biological Chemistry 263 45764585

Welsh AO and Enders AC (1985) Light and electron microscopic examination of the mature decidual cells of the rat with emphasis on the antimesometrial decidua and its degeneration American Journal of Anatomy 172 1-29

Winterhager E, Grümmer R, Jahn E, Willecke K and Traub O (1993) Spatial and temporal expression of connexin 26 and connexin 43 in rat endometrium during trophoblast invasion Developmental Biology 157 399-409

Wood C, Kabat EA, Murphy LA and Goldstein IJ (1979) Immunochemical studies of the combining sites of two isolectins, $\mathrm{A}_{4}$ and $\mathrm{B}_{4}$, isolated from Bandeiraea simplicifolia. Archives of Biochemistry and Biophysics 198 1-11 